\title{
High-resolution urban air quality monitoring using sentinel satellite images and low-cost ground-based sensor networks
}

\author{
Joseph Gitahi ${ }^{1}$, Michael Hahn \\ Hochschule für Technik Stuttgart, Schellingstrasse 24, D-70174 Stuttgart, Germany
}

\begin{abstract}
Satellite remote sensing aerosol monitoring products are readily available but limited to regional and global scales due to low spatial resolutions making them unsuitable for citylevel monitoring. Freely available satellite images such as Sentinel -2 at relatively high spatial $(10 \mathrm{~m})$ and temporal (5 days) resolutions offer the chance to map aerosol distribution at local scales. In the first stage of this study, we retrieve Aerosol Optical Depth (AOD) from Sentinel 2 imagery for the Munich region and assess the accuracy against ground AOD measurements obtained from two Aerosol Robotic Network (AERONET) stations. Sen2Cor, iCOR and MAJA algorithms which retrieve AOD using Look-up-Tables (LUT) pre-calculated using radiative transfer (RT) equations and SARA algorithm that applies RT equations directly to satellite images were used in the study. Sen2Cor, iCOR and MAJA retrieved AOD at 550nm show strong consistency with AERONET measurements with average correlation coefficients of $0.91,0.89$ and 0.73 respectively. However, MAJA algorithm gives better and detailed variations of AOD at $10 \mathrm{~m}$ spatial resolution which is suitable for identifying varying aerosol conditions over urban environments at a local scale. In the second stage, we performed multiple linear regression to estimate surface Particulate Matter $\left(\mathrm{PM}_{2.5}\right)$ concentrations using the satellite retrieved AOD and meteorological data as independent variables and ground-measured $\mathrm{PM}_{2.5}$ data as the dependent variable. The predicted $\mathrm{PM}_{2.5}$ concentrations exhibited agreement with ground measurements, with an overall coefficient $\left(\mathrm{R}^{2}\right)$ of 0.59 .
\end{abstract}

\section{INTRODUCTION}

The study of aerosol concentrations in the atmosphere is essential due to the role they play in Earth's climate. The aerosols which could be either natural or anthropogenic absorb or scatter sunlight with an impact on the Earth's energy budget. Subtypes of atmospheric particles, the particulate matter (PM), are under criticism for their adverse effects on human health. Health effects include damage to respiratory and cardiovascular systems with $\mathrm{PM}_{2.5}$ posing higher risks owing to their smaller size. The risk is higher in urban regions where an estimated $55 \%$ of the World's population live [1] which is as a result of higher anthropogenic aerosols emissions from transport, industries, power plants and household sources.

Satellite remote sensing has been used to determine the aerosol concentration in the atmosphere based on the inversion of radiative transfer (RT) equations which model the scattering and absorption of solar radiation by aerosols, gas and water molecules in the atmosphere. The retrieval of columnar aerosol optical depth/thickness (AOD/AOT) forms a crucial step in the atmospheric correction of satellite images to generate surface reflectance products. By using the top-of-atmosphere (TOA) reflectance received by the satellite sensors and known surface reflectances/ bottom-of-atmosphere (BOA), the optical thickness of the atmosphere can be determined. The RT equations generate Look-up-Tables (LUT) which model the relationship between TOA reflectances and $\mathrm{AOD}$ in the required electromagnetic spectrum range. TOA reflectances follow the RT equation as expressed in Equation 1.

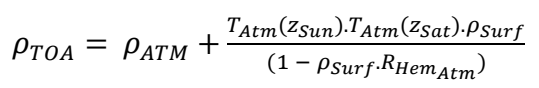

Where $\rho_{T O A}$ is the TOA reflectance, $\rho_{A T M}$ is the atmospheric reflectance, $\rho_{\text {Surf }}$ is the surface reflectance, $R_{\text {Hem }}$ Atm is the hemispheric albedo of the atmosphere, $T_{A t m}\left(z_{\text {Sun }}\right)$ and $T_{A t m}\left(z_{\text {Sat }}\right)$ are transmissions of the

This paper is an extended version of our paper, Gitahi, J., Hahn, M., Ramirez, A.: High-resolution Urban Aerosol Monitoring Using Sentinel-2 Satellite Images, Vol. 3, No. 1, pp. 102-111. doi:10.22059/eoge.2019.289985.1063; https://eoge.ut.ac.ir/article_72963.html. In the present paper we extend our work to include Particulate Matter (PM) estimation from satellite retrieved Aerosol Optical Depth (AOD).

* Corresponding author: joseph.gitahi@hft-stuttgart.de 
atmosphere for sun $z_{\text {Sun }}$ and satellite $z_{\text {Sat }}$ zenith distances respectively.

AOD is then obtained by subtracting Rayleigh scattering and gaseous absorption influences from the total optical thickness of the atmosphere as shown in Equation 2.

$$
\delta_{A e r}(\lambda)=\delta(\lambda)-\delta_{\text {Gas }}(\lambda)-\delta_{\text {Ray }}(\lambda)
$$

Where $\delta$ is the optical thickness of the atmosphere at wavelength $\lambda, \delta_{\text {Aer }}(\lambda)$ the $\mathrm{AOD}, \delta_{G a s}(\lambda)$ the optical thickness of gaseous absorbers and $\delta_{\text {Ray }}(\lambda)$ the optical thickness of Rayleigh Scattering.

One of the algorithms used to estimate AOD is the Dark Object Subtraction (DOS) or Dark Target (DT) method which relies on the presence of dark pixels in an image. For these dark pixels of zero or minimal surface reflectance, TOA reflectances received are a result of atmospheric reflectances $\rho_{T O A}=\rho_{A T M}$. The estimated atmospheric reflectance is subtracted from the entire image scene to calculate the surface reflectance and used in RT models to retrieve the AOD. The assumption of this method is that dark pixels of known reflectances are found in an image scene and that the atmosphere is spatially uniform over the image.

The second approach is the multispectral method which relies on the known constant spectral properties of dark dense vegetation (DDV) in the electromagnetic spectrum [2,3]. It uses the empirical relationships between surface reflectances in the blue and red bands with the SWIR band $\rho$ Blue $=0.5 * \rho S W I R$ and $\rho$ Red $=0.25 * \rho S W I R$. This is based on the spectral properties of healthy vegetation where chlorophyll strongly absorbs light at blue and red bands and the moisture content absorption peaks in the SWIR band. The DDV algorithm has been used extensively to retrieve AOD over land surfaces with high precision from images acquired by Moderate Resolution Imaging Spectrometer (MODIS) on board Terra and Aqua satellites. The assumptions of this method are that pixels with low BOA reflectances in the visible spectrum are present such as dark vegetation or water bodies and that the ratio between BOA reflectances at different wavelengths is constant [4]. The application of this approach for AOD retrievals is limited over brighter surfaces and arid regions which have sparse or no vegetation. This approach is also limited by locational, seasonal and angular dependence of visible and SWIR bands surface ratios [5]. Deep Blue (DB) algorithm is used for AOD retrievals over brighter surfaces especially arid and semi-arid regions where there are no dark pixels for the DDV method. In MODIS, this algorithm uses the $412 \mathrm{~nm}$ band also referred to as the "deep blue" band in which aerosols appear bright and surfaces darker unlike in visible bands where the contrast between aerosols and the surfaces is not easily distinguishable.

AOD can also be retrieved using the multi-temporal method which is based on the assumption that land surfaces change slowly over time and remain relatively constant especially with short satellite revisit times and a constant viewing angles [5]. The changes in reflectances observed from one image to another of the same scene can thus be attributed to the atmospheric aerosols which vary rapidly over time. Based on these assumptions, the approach is less accurate for satellites with longer revisit times and when vegetation in a scene changes rapidly in between acquisitions. The method's sensitivity to aerosols variation also decreases and the error increases with increasing surfaces reflectances [6].

Readily available satellite AOD products like the Moderate Resolution Imaging Spectroradiometer (MODIS) product MOD04 at 550nm provides a high temporal resolution for daily based monitoring at $3 \mathrm{~km}$ (MOD04_3K) and 10km (MOD04_L2) spatial resolutions suited for global and regional scales but incapable of detailed urban air quality monitoring [7]. Both DT and DB algorithms are used for AOD retrieval and they both rely on darker surfaces to estimate the atmosphere's contribution to TOA radiances but differ in the way they account for surface reflectance. The DT algorithm uses dark surfaces in two visible channels $470 \mathrm{~nm}$ and $660 \mathrm{~nm}$ and the approximate transparency of the atmosphere at $2120 \mathrm{~nm}$ to obtain an accurate estimation of the atmospheric scattering which works best over dark vegetation and water bodies. The DB algorithm retrieves AOD using dark surfaces in two blue channels $412 \mathrm{~nm}$ and $470 \mathrm{~nm}$ and little absorption by dust in a red channel $670 \mathrm{~nm}$ and addresses the issue of AOD retrievals over bright land surfaces [8].

European Space Agency's (ESA) Copernicus programme provides aerosol products from their Sentinel-3 and Sentinel-5P satellite missions. The Sentinel-3 AOD product at 550nm, available since October 2018, has a 300m spatial resolution, the Aerosol Index (AI) from Sentinel-5P a spatial resolution of $7 \mathrm{~km}$ $x 3.5 \mathrm{~km}$. The Sentinel-3 SYN AOD is one of the synergy products derived from the Ocean and Land Color Instrument (OLCI) and Sea and the Land Surface Temperature Radiometer (SLSTR) sensors. The Sentinel -3 SYN algorithm used to estimate aerosols and surface reflectances uses a synergistic approach to retrieve AOD by combining DOS and multi-view-angle (MVA) methods [9]. The DOS method is used for single view AOD retrievals using a correlation of BOA reflectances from known surfaces of dark vegetation and water bodies. Dark pixels are identified by calculating the Normalized Difference Vegetation Index (NDVI) using reflectances in the infrared band $(870 \mathrm{~nm})$ and the red band (670) Equation 3.

$$
N D V I=\frac{\rho_{870}-\rho_{670}}{\rho_{870}+\rho_{670}}
$$

The multi-view-angle method assumes a constant angular variation of BOA reflectances across a wavelength which is as a result of Bidirectional Reflectance Distribution Function (BRDF). For the SLSTR sensor, the ratio of BOA reflectances at nadir and off-nadir viewing angles is well correlated between bands. The advantage of this approach over the DOS is that it does not require prior information of $\mathrm{BOA}$ 
reflectances. The AOD retrieved using the MVA technique exhibits high consistency with groundmeasured AERONET AOD with a correlation coefficient of 0.87 for mixed land surfaces.

On the ground, Aerosol Robotic Network (AERONET) stations form a global network of sun photometers measuring AOD and aerosol properties more accurately and are used for validation and calibration of satellite AOD retrievals. However, these stations are sparsely distributed globally and hence the need for satellite retrieved AOD for better coverage.

Compared to ground-based aerosol monitoring sensors, satellite remote sensing has inherent advantages since the spatial coverage is nearly continuous over large extents and provides high spatial resolution data. However, the sparsely distributed ground-based sensors like AERONET provide the most accurate AOD measurements and are thus considered as ground truth. The goal of this study is to assess the suitability of Sentinel-2 images to retrieve AOD at a higher spatial resolution capable of mapping spatial and temporal variability of aerosols at city-levels. Different AOD retrieval algorithms were used and evaluated for accuracy against AERONET data.

\section{METHODOLOGY}

\section{Study Areas}

The first study area is the Munich region which has mixed urban and rural land surfaces. The study area has three AERONET stations, two of which are operational. The AOD measurements from the ground stations are crucial in assessing the performance of AOD retrieval algorithms. The locations of the two stations, Munich University $(521 \mathrm{~m}$ asl) in the middle of the city and HohenpeissenbergDWD $(956 \mathrm{~m}$ asl) in a rural region of which the land cover is predominantly vegetated, offer the opportunity to assess the suitability of the AOD retrieval algorithms in both land use cases.

The city region lies on elevated plains at an average elevation of $520 \mathrm{~m}$ above sea level, rising gradually towards the Bavarian Alps located approximately $50 \mathrm{~km}$ in the south. The estimated population of the city is 1.45 million inhabitants. The city region has major motorways with intense traffic and light industries are spread out across the city.

The Stuttgart city region was used as the second study area for estimating $\mathrm{PM}_{2.5}$ from the retrieved AOD. In Stuttgart, air pollution levels have in the past exceeded the thresholds set by WHO which can be attributed to the high traffic and industrial activities. Geographically, the city is located in a valley basin surrounded by mountains which inhibit airflow further worsening the air quality situation.

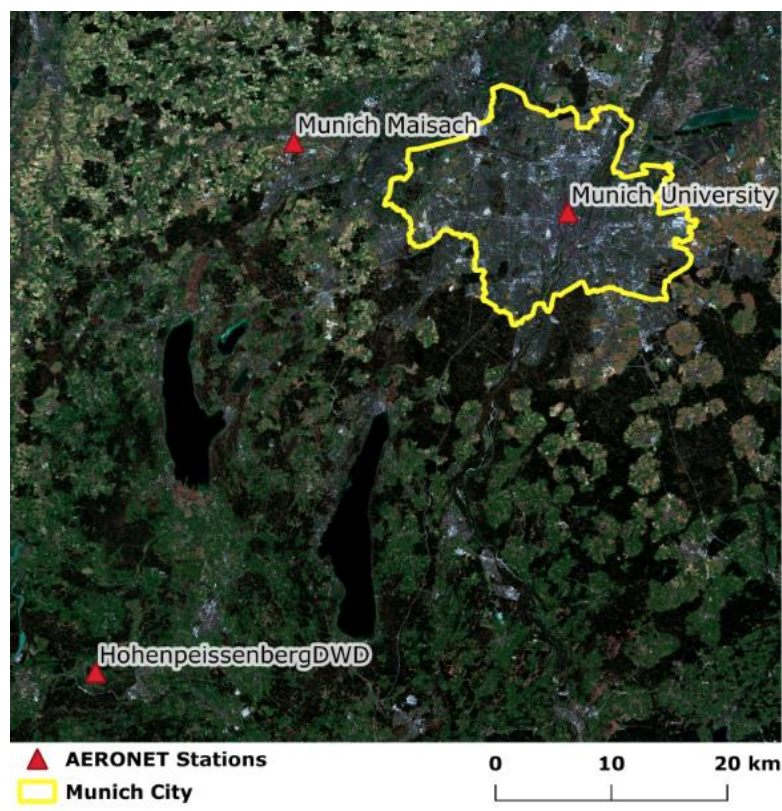

Figure 1: Sentinel -2 satellite image of the Munich city region study area, showing the region boundary and the three AERONET stations

\section{Data}

To evaluate different AOD retrieval methods, we used Sentinel $-2 \mathrm{~A}$ and $2 \mathrm{~B}$ images of the study region acquired between January and October 2018. In total 43 clear images which had $30 \%$ cloud cover or less were selected for the study. Corresponding AERONET cloud-screened and quality controlled Level 1.5 AOD data from two stations in the study area Munich University and HohenpeissenbergDWD was used for validating the satellite retrieved AOD [10]. Data from a third AERONET station, Munich Maisach, was not available for the study period. Since all the AOD retrievals are calculated at $550 \mathrm{~nm}$ wavelength, AERONET AOD at $500 \mathrm{~nm}$ was interpolated using the Angstrom power-law Equation 4. Aerosol properties, single scattering albedo (SSA) and asymmetric factor from the stations, were linearly interpolated to $550 \mathrm{~nm}$ wavelength and used to retrieve $\mathrm{AOD}$ in section 0 .

$$
A O D_{550 \mathrm{~nm}}=A O D_{500 \mathrm{~nm}}\left(\frac{550}{500}\right)^{-\alpha}
$$

Where $\alpha$ is the Angstrom exponent 440-870nm.

In the second stage of the study, $\mathrm{PM}_{2.5}$ estimation in Stuttgart city, we used $\mathrm{PM}_{10}$ and $\mathrm{PM}_{2.5}$ measurements from Luftdaten low-cost sensors network, a citizen science project by OK Lab Stuttgart. This dense network with approximately 250 sensors offers relative air quality information but at lower accuracies compared to air monitoring stations operated by the state regulatory agency. Meteorological parameters, temperature, humidity, atmospheric pressure, wind direction and speed are obtained from OpenWeatherMap service which has 23 stations in the area. In total, PM and weather data from six days is aggregated to hourly averages for use in AOD$\mathrm{PM}_{2.5}$ analysis. 


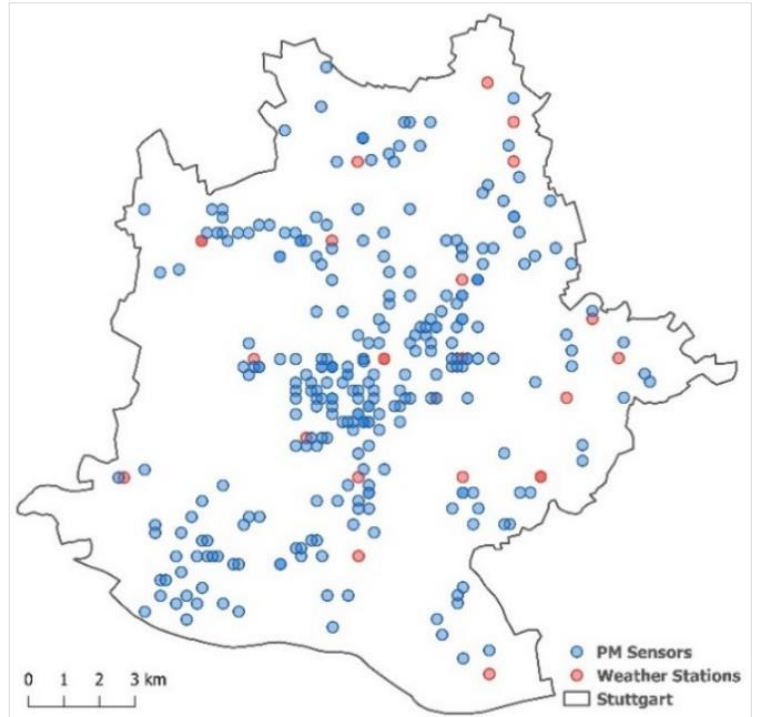

Figure 2: A map of Stuttgart city showing the distribution of Luftdaten PM sensors and weather stations.

\section{AOD Retrieval Algorithms}

\section{Sen2Cor}

ESA's Copernicus programme provides Sentinel -2 images either as TOA Level-1C or BOA Level-2A products. For the Level-1C products, Sen2Cor processor is freely available for users to perform single-date atmospheric corrections. AOD, water vapour and scene classification bands are created as by-products of the correction process. The by-products are also available readily in Level-2A products. The tool is available either as a standalone or a plugin to ESA's Sentinel Application Platform (SNAP) software. AOD is derived at $550 \mathrm{~nm}$ using the DDV algorithm based on the SWIR (band 12) and the visible bands red (band 4) and blue (band 2). It requires the presence of dark vegetation, dark soils or water surfaces in an image scene. In the absence of dark pixels, atmospheric correction is performed with a constant AOD specified by a start visibility value at $40 \mathrm{~km}$ corresponding to an AOD of 0.2 at sea level [1113]. This was not an issue as our study region contains densely vegetated areas and water bodies as shown in Figure 1. The Level-1C images were processed to Level$2 \mathrm{~A}$ at $20 \mathrm{~m}$ resolution using SNAP and a factor of 0.001 applied to the aerosol band digital numbers to derive the AOD values.

\section{MACCS-ATCOR Joint Algorithm (MAJA)}

MAJA is an atmospheric correction algorithm combining modules from Multi-Temporal Atmospheric Correction and Cloud Screening (MACCS) developed by CNES and CESBIO and Atmospheric Correction (ATCOR) by DLR [5]. The algorithm, applicable to Landsat -8, Sentinel -2, VEN $\mu$ S and Formosat-2 satellite images, employs different techniques to estimate aerosols for the atmospheric correction process. Using the multitemporal method, for two consecutive cloud-free satellite observations at dates $D$ and $D_{T}$, the algorithm searches for AODs of both dates that minimize the squared differences between the corrected surface reflectances Equation 7.

The setback to this method is that the accuracy is reduced when the AOD is nearly similar for both dates making it impossible to retrieve an absolute AOD value. To improve the AOD retrievals in this scenario, the present image is compared to a reference image from an older iteration of the algorithm in Equation 8. A cost function Equation 5 which is a sum of Equations 7 and 8 errors, and a Levenberg-Marquardt non-linear least mean squares (LMS) algorithm searches for the AOD of $D$ and $D_{T}$ that minimizes the error.

$\operatorname{cost}_{M T}=\sum_{\text {validpixels }} \operatorname{err}_{M T}^{2}$

Where:

$\operatorname{err}_{M T}^{2}=\left(K_{1}^{2} \operatorname{err}_{1}^{2}+K_{2}^{2} \operatorname{err}_{2}^{2}\right)$

$\operatorname{err}_{1}=a t_{c o r}\left(\rho_{T O A}(D), \tau\right)-a t_{c o r}\left(\rho_{T O A}\left(D_{T}\right), \tau_{T}\right)$

$\operatorname{err}_{2}=a t_{c o r}\left(\rho_{T O A}(D), \tau\right)-\left(\rho_{S u r f}\left(D_{T}\right)\right)$

Where $a t_{c o r}$ is the atmospheric correction function linking TOA reflectances to their corresponding BOA reflectances for a given AOD $\tau$ and aerosol model, $\rho_{\text {Surf }}\left(D_{T}\right)$ is BOA reflectance from the reference image. The reflectances used in the cost function are from the blue band range which has a lower temporal variation. $K_{1}$ and $K_{2}$ weight the contribution of $\operatorname{err}_{1}$ and $e r r_{2}$ where $K_{2}$ is set to one and $K_{1}$ is proportional to the mean value of the difference of TOA reflectances from dates $D$ and $D_{T}$.

The second technique used is the multispectral method which is based on the DDV approach but relying on blue-red bands relationships instead of the visible /SWIR relationships. This adapted method seeks to address issues affecting the DDV approach which are locational, seasonal and angular dependences of visible/SWIR surface ratio. Due to the errors observed when SWIR band is used, the algorithm uses a more robust blue-red relationship which is established for each of the satellite sensors applicable to diverse locations and seasons Equation 9.

$\rho_{\text {blue }}^{\text {surf }}=C * \rho_{\text {red }}^{\text {surf }}$

Where: $C$ is the blue-red coefficient. For Sentinel -2 $C=0.45, \mathrm{~B} 1$ is used as the blue band and B4 is the red band.

Since only one equation is used, retrieving both the $\mathrm{AOD}$ and the aerosol model is not possible and a constant 
aerosol model for any given location has to be used. This assumption of a constant aerosol model introduces atmospheric correction errors. MAJA's multispectral method uses a cost function in Equation 10 by summing up the squares of differences between blue surface reflectances after atmospheric correction and the blue surface reflectances predicted from the red band.

$\operatorname{cost}_{M S}=\sum_{\text {validpixels }} K^{2} \times \operatorname{err}_{M S}^{2}$

Where:

$\operatorname{err}_{M S}^{2}=a t_{c o r}\left(\rho_{\text {blue }}^{T O A}(D), \tau\right)-\left(A \times a t_{c o r}\left(\rho_{\text {red }}^{T O A}(D), \tau\right)+B\right)$

Where the weight $K$ is equal to the NDVI to account for better correlation of the blue-red relationship for high NDVI, $A$ and $B$ are gains linking the blue and red BOA reflectances for a particular satellite sensor.

The algorithm uses a third technique which combines the multi-temporal and multispectral algorithms and relies on minimizing the cost function using the LMS algorithm. The cost function combines the multitemporal and multispectral Equations 6 and 11 respectively shown in Equation 12.

cost $=\sum_{M T \text { validpixels }} K_{M T}^{2}$ err $_{M T}^{2}+\sum_{M S \text { salidpixels }} K_{M S}^{2}$ err $_{M S}^{2}$

Where $K_{M T}$ and $K_{M S}$ are weighting coefficients for the contribution of the multitemporal and multispectral methods. $K_{M T}$ takes into account the time interval between two consecutive images, whose increase leads to decreased AOD retrieval accuracy due to surfaces changing over time.

For the three approaches, two constraints, a lower and an upper boundary, are applied to limit errors in AOD estimates. The lower boundary being that AOD cannot be negative and the upper boundary estimated using the Dark Object Subtraction (DOS) method. For AOD retrieval, clear pixels with the minimum reflectance in the blue band are selected and checked in previous images to avoid undetected cloud shadows. The two bounds obtained are then added to the cost function. To address the need for constant viewing angles, directional correction is performed in the multi-temporal approach to enable merging a time series of images acquired from different orbits which sometimes is the case with overlapping Sentinel-2 images. This also helps in densifying the time-series. Finally, interpolation of missing AOD retrievals due to masked pixels and smoothing of the image is done to deliver the final output.

The publicly available MAJA code (V3.3) [6] was run in a Linux environment. Earlier versions of MAJA used a continental aerosol model to generate LUTs for atmospheric correction, however starting with version 3.1 , the algorithm uses CAMS aerosol products to model the aerosol type. Starting with version 3.2, CAMS data is not only used to estimate the aerosol type but also optionally as the default value for AOD estimates. The
AOD is used with low weight in the cost function which leads to greater influence where the algorithm does not find sufficient suitable pixels for estimation and has no influence in case of good AOD estimates. To run MAJA with CAMS option, we downloaded CAMS data from January to October. SRTM DTM and water body datasets for the study area were prepared using the pre-processing scripts provided. The output of MAJA algorithm is in MACCS format and contains the aerosols band in the atmospheric and biophysical (ATB) parameters file as band number 2 at $10 \mathrm{~m}$ spatial resolution. The final postprocessing step to obtain the AOD is multiplying by the quantification value 0.005 .

\section{iCOR}

iCOR is an atmospheric correction algorithm for both land and water developed by Vlaams Instituut voor Technologisch Onderzoek (VITO) Remote Sensing Unit provided freely as a SNAP plugin for Landsat-8 and Sentinel-2 data [14]. AOD is retrieved using the method by [15] which is then used as an input to the MODTRAN5 radiative transfer equation used for correcting atmospheric effects. The first step in correcting Sentinel-2 images is water and cloud pixels masking performed using a single band (NIR B8) thresholding for water and multiple threshold levels for the cloud pixels. The algorithm also takes advantage of the present SWIRCirrus band (B10) to improve detection of cirrus clouds. The TOA scene is then partitioned into $15 \times 15 \mathrm{~km}$ tiles which are assumed to include high spectral variation and atmospherically homogeneous.

In each of the tiles, the lowest radiance value for each band is selected as the dark target spectrum and the approximate path radiance retrieved using pre-calculated MODTRAN5 look-up-table (LUT). The AOD value leading to the path radiance closest to the dark spectrum is considered the upper boundary for the specific tile. Five reference pixels with high spectral contrast are selected based on their NDVI values from TOA reflectances and used in the next step to refine the estimated AOD through an endmember inversion technique. The technique models surface reflectance of the five pixels as a linear combination of two predefined pure green vegetation and bare soil spectra that act as artificial endmembers Equation 13.

$\rho_{s}=C_{v} * \rho_{v e g}+C_{s} * \rho_{\text {soil }}$

Where: $\rho_{s}, \rho_{v e g}, \rho_{\text {soil }}$ are surface reflectances of reference pixels, predefined vegetation and soil spectra, $\mathrm{C}_{\mathrm{v}}$ and $C_{s}$ are independent coefficients weighting vegetation and soil proportions $C_{v}$ and $\mathrm{C}_{\mathrm{s}}$ are free in the inversion resulting in 11 parameters as degrees of freedom; two parameters for each of the five pixels and AOD. The inversion is performed through the minimisation of the Merit function $\delta^{2}$ Equation 14 [16].

$\delta^{2}=\sum_{1}^{N} \omega_{p i x} \sum_{\lambda_{i}} \frac{1}{\lambda_{i}^{2}}\left[L_{p i x, \lambda_{i}}^{S I M}-L_{p i x, \lambda_{i}}^{S E N S}\right]^{2}$ 
Where: $L^{S I M}$ is simulated TOA radiance from MODTRAN5 LUT, $L^{S E N S}$ is measured TOA radiance, $\lambda_{i}$ is centre wavelength of the ii-th band and $\omega_{\text {pix }}$ is weighting factor (2.0 for pure vegetation, 1.5 for mixed and 1.0 for pure soil pixels)

The AOD value leading to the minimum $\delta^{2}$ value in the inversion is selected for the cell. The last steps in the retrieval process are an interpolation of the missing pixels due to cloud masking and smoothing of the mosaiced tiles. The iCOR algorithm avoids the need for dark targets making it less restrictive than the DDV approach. Atmospheric correction was performed on Sentinel -2 L1C images and the AOD intermediate band at $60 \mathrm{~m}$ spatial resolution exported for analysis.

\section{Simplified high-resolution MODIS Aerosol Retrieval Algorithm (SARA) \\ The Simplified high-resolution MODIS Aerosol} Retrieval Algorithm (SARA) [17] has been used to retrieve AOD from MODIS images at $500 \mathrm{~m}$ spatial resolution. The inputs of the algorithm are the TOA and BOA reflectances, sun and view angles, and aerosol properties obtained from AERONET ground stations. The algorithm is considered simplified as it does not require LUTs to retrieve AOD. While the other three algorithms depend on LUTs pre-computed using RT equations to model the relationship between TOA reflectances and AOD, in SARA, the RT equation is applied directly together with aerosol properties derived from AERONET stations. The algorithm assumes Lambertian surfaces, single scattering approximation, and that single scattering albedo and asymmetry factors are constant over a region at a particular time.

Processing using this algorithm was implemented in Python to retrieve AOD at 550nm. From Sentinel-2 Level 1C images, TOA reflectances for band $3(560 \mathrm{~nm})$, sun and view azimuth and zenith angles were extracted as grids. BOA reflectances were obtained from band 3 of Level 2A images. The images were all resampled to $20 \mathrm{~m}$ resolution and clipped to a smaller region of the study area for computational reasons. To mask out clouds, cloud shadows, water and snow pixels, the FMASK algorithm was used which offers better discrimination of non-clear pixels compared to the mask bands in Level 2A products $[18,19]$. SRTM DTM of the study region was obtained and resampled to $20 \mathrm{~m}$ for calculations in the next steps.

In SARA algorithm, the AOD $\tau_{a}$ retrieval relies on aerosol reflectance in the absence of air molecules

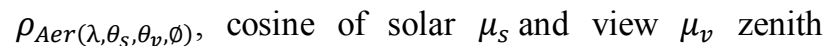
angles, single scattering albedo $\omega_{0}$ and aerosol scattering phase function $P_{a\left(\theta_{s}, \theta_{v}, \emptyset\right)}$ as given in Equation 15.

$\tau_{a, \lambda}=\frac{4 \mu_{s} \mu_{v}}{\omega_{0} P_{a\left(\theta_{s}, \theta_{v}, \varnothing\right)}} \rho_{A \operatorname{er}\left(\lambda, \theta_{s}, \theta_{v}, \emptyset\right)}$

The aerosol scattering phase function gives the angular dependence of light scattered by aerosols and is calculated using Equation 16 [20].
$P_{a\left(\theta_{s}, \theta_{v}, \emptyset\right)}=\frac{1-g^{2}}{\left[1+g^{2}-2 g \cos (\pi-\Theta)\right]^{\frac{3}{2}}}$

Where $g$ is the asymmetry parameter and $\Theta$ is the scattering phase angle defined in Equation 17, a function of solar zenith angle $\theta_{s}$, view zenith angle $\theta_{v}$ and relative azimuth angle $\varnothing=\emptyset_{s}-\emptyset_{v}$.

$\Theta=\cos ^{-1}\left(-\cos \theta_{s} \cos \theta_{v}+\sin \theta_{S} \sin \theta_{v} \cos \emptyset\right)$

The aerosol reflectance $\rho_{\operatorname{Aer}\left(\lambda, \theta_{s}, \theta_{v}, \varnothing\right)}$ is calculated by subtracting the Rayleigh path reflectance $\rho_{\operatorname{Ray}\left(\lambda, \theta_{s}, \theta_{v}, \varnothing\right)}$ and the surface function from the satellite measured TOA reflectances as shown in Equation 18 [21].

$$
\begin{gathered}
\rho_{\operatorname{Aer}\left(\lambda, \theta_{s}, \theta_{v}, \emptyset\right)}=\rho_{T O A\left(\lambda, \theta_{s}, \theta_{v}, \emptyset\right)}-\rho_{R a y\left(\lambda, \theta_{s}, \theta_{v}, \emptyset\right)} \\
-\frac{T_{\left(\theta_{s}\right)} T_{\left(\theta_{v}\right)} \rho_{S\left(\lambda, \theta_{s}, \theta_{v}, \emptyset\right)}}{1-\rho_{S\left(\lambda, \theta_{s}, \theta_{v}, \emptyset\right)} S_{(\lambda)}}
\end{gathered}
$$

Where $\rho_{s\left(\lambda, \theta_{s}, \theta_{v}, \emptyset\right)}$ is the BOA reflectance, $S_{(\lambda)}$ is the atmospheric backscattering, $T_{\left(\theta_{S}\right)}$ and $T_{\left(\theta_{v}\right)}$ are the transmissions of the atmosphere on the sun-surface and surface-sensor paths respectively. TOA $\rho_{T O A\left(\lambda, \theta_{s}, \theta_{v}, \varnothing\right)}$ and BOA $\rho_{s\left(\lambda, \theta_{s}, \theta_{v}, \varnothing\right)}$ reflectances were obtained directly from Sentinel-2 Level $1 \mathrm{C}$ and Level $2 \mathrm{~A}$ products respectively. Rayleigh path reflectance $\rho_{\operatorname{Ray}\left(\lambda, \theta_{s}, \theta_{v}, \emptyset\right)}$ calculation is based on the Rayleigh optical depth and the sun and view zenith angles, as expressed in Equation 21.

$T_{\left(\theta_{s}\right)}=\exp \left[-\left(\tau_{R}+\tau_{a}\right) * 1 / \mu_{s}\right]$

$T_{\left(\theta_{v}\right)}=\exp \left[-\left(\tau_{R}+\tau_{a}\right) * 1 / \mu_{v}\right]$

$\rho_{R a y}\left(\lambda, \theta_{s}, \theta_{v}, \emptyset\right)=\frac{w_{R} \tau_{R} P_{R\left(\lambda, \theta_{s}, \theta_{v}, \emptyset\right)}}{\mu_{s} \mu_{v}}$

$S_{(\lambda)}=\left(0.92 \tau_{R}+(1-g) \tau_{a}\right) e^{-\left(\tau_{R}+\tau_{a}\right)}$

Where $\tau_{R}$ is the Rayleigh optical depth as expressed by Equation 23 in the visible range spectrum [22].

$\tau_{R}(\lambda)=\frac{p_{z}}{p_{0}} 0.00877 \lambda^{-4.05}$

The ambient pressure $p_{z}$ is calculated with respect to surface elevation using the Barometric formula in Equation 24 [23].

$p_{z}=p_{0} e^{\frac{M g r}{R T} z}$

Where $p_{0}$ is the standard atmospheric pressure at sea level, $M$ is the Molar mass of dry air, $g r$ the EarthSurface gravitational acceleration, $R$ the universal gas constant, $T$ the standard temperature and $z$ the elevation obtained from SRTM DTM. 
$w_{R}$ is the Rayleigh single-scattering albedo approximated to 1 and $P_{R}\left(\lambda, \theta_{s}, \theta_{v}, \emptyset\right)$ Is the Rayleigh scattering phase function [24].

$$
P_{R\left(\lambda, \theta_{S}, \theta_{v}, \emptyset\right)}=\frac{3}{4}\left(1+\cos ^{2}(\Theta)\right)
$$

Thus, based on Equation 15, all required parameters for AOD retrieval can be estimated with only three unknowns, single scattering albedo $\omega_{0}$, asymmetry parameter $g$ and the AOD $\tau_{a}$ itself can be retrieved as in shown in Equation 26 [17].

$$
\begin{aligned}
& \tau_{a, \lambda}=\frac{4 \mu_{s} \mu_{v}}{\omega_{0} \rho_{a\left(\theta_{s}, \theta_{v}, \emptyset\right)}} \\
& {\left[-\frac{\rho_{T O A\left(\lambda, \theta_{s}, \theta_{v}, \emptyset\right)-} \rho_{R a y\left(\lambda, \theta_{S}, \theta_{v}, \emptyset\right)} e^{-\frac{\tau_{R}+\tau_{a, \lambda}}{\mu_{S}}} e^{-\frac{\tau_{R}+\tau_{a, \lambda}}{\mu_{v}}} \rho_{s\left(\lambda, \theta_{S}, \theta_{v}, \emptyset\right)}}{1-\rho_{s\left(\lambda, \theta_{s}, \theta_{v}, \emptyset\right)}\left(0.92 \tau_{R}+(1-g) \tau_{a, \lambda}\right) e\left[-\left(\tau_{R}+\tau_{a, \lambda}\right)\right]}\right]}
\end{aligned}
$$

Single scattering albedo $\omega_{0}$ and asymmetry parameter $g$ were obtained from the two AERONET stations. Using the values measured in $441 \mathrm{~nm}$ and $676 \mathrm{~nm}$ wavelengths, $\omega_{0}$ and $g$ which were linearly interpolated to $550 \mathrm{~nm}$. The equation was solved using Scipy's fsolve module in Python.

\section{AOD-PM ${ }_{2.5}$ estimation}

Due to the full coverage of satellite images, retrieved AOD can be utilized to estimate $\mathrm{PM}_{2.5}$ concentrations at full spatial extents based on the correlation between AOD and $\mathrm{PM}_{2.5}$. The relationship between the columnar AOD and the surface level $\mathrm{PM}_{2.5}$ concentration is heavily influenced by meteorological conditions. In previous studies, temperatures have been observed to have a negative correlation with PM concentrations while relative humidity shows positive correlations [25]. Relative humidity promotes the deposition of PM as moisture particles adhere to PM leading to higher concentrations. Temperature and atmospheric pressure influence the transport and accumulation of PM by affecting convection while winds lead to PM dispersion $[26,27]$.

In this study, a multiple linear regression model used to estimate $\mathrm{PM}_{2.5}$ with Sentinel -3 SYN AOD and meteorological data. The meteorological data was fused with $\mathrm{PM}_{2.5}$ measurements using spatial joins on the closest weather station and the AOD pixel values extracted for each PM sensor. The null values due to missing pixels in AOD images and missing parameters from the weather stations recordings were deleted from the dataset. Erroneous $\mathrm{PM}_{2.5}$ recordings attributed to faulty sensors and outliers were also excluded from the regression analysis.

\section{RESULT ANALYSIS}

\section{Statistical Analysis}

Evaluation of the satellite retrieved AODs compared with AOD measured by Munich University and HohenpeissenbergDWD AERONET stations was performed using three statistical indicators. The correlation coefficient $(\mathrm{R})$ was used as a measure of consistency between AOD retrieved using the different algorithms and AERONET AOD at 550nm. The rootmean-square error (RMSE) was used as a measure of the AOD differences and the mean absolute error (MAE) as a measure of the error magnitude

Table 1. Since aerosols exhibit high temporal variation, statistical analysis was performed on AODs within \pm 15 minutes between satellite images acquisitions and the AERONET measurements. Where AERONET data was missing (date 27.04.2018 for Munich University station and 24.09.2019 for HohenpeissenbergDWD station), the dates were also excluded from the analysis.

From the results in

Table 1, AOD retrieved using the Sen2Cor algorithm shows better consistency with the AERONET AOD and the lowest error magnitudes for both stations. The study area has sufficient dark pixels from the water bodies and dense vegetation which is required for the DDV multispectral AOD retrieval approach. For the Munich University station, satellite retrieved AOD for the three algorithms shows strong agreement with the AERONET AOD. However, the consistency is reduced for HohenpeissenbergDWD station with MAJA algorithm showing significantly lower agreement levels. HohenpeissenbergDWD station is located in an area predominantly containing vegetation, which means the land surface reflectance (LSR) varies greatly over time compared to the urban region where Munich University station is located. This high variance in LSR over time results to less accurate AOD retrievals and hence the lower agreement levels when compared to AERONET AOD. Figure 3 shows the scatterplots and regression slopes of the satellite AOD retrievals and AERONET AOD over the two stations.

Table 1: Comparison between Sentinel -2 satellite retrieved

\begin{tabular}{|c|c|c|c|c|c|}
\hline & $\mathbf{R}$ & RMSE & MAE & $\mathbf{N}$ & Regression Line \\
\hline Sen2Cor & 0.96 & 0.004 & 0.049 & 27 & $\begin{array}{l}Y= \\
0.035\end{array}$ \\
\hline iCOR & 0.92 & 0.005 & 0.056 & 24 & $\begin{array}{l}\mathrm{Y}=1.055 \mathrm{x}- \\
0.051\end{array}$ \\
\hline МАJA & 0.81 & 0.006 & 0.054 & 27 & $\begin{array}{l}Y= \\
0.003\end{array}$ \\
\hline
\end{tabular}
AOD and AERONET AOD at 550nm.

Munich University Station

\begin{tabular}{|c|c|c|c|c|c|}
\hline & $\mathbf{R}$ & RMSE & MAE & $\mathbf{N}$ & Regression Line \\
\hline Sen2Cor & 0.86 & 0.003 & 0.035 & 31 & $\begin{array}{l}Y=0.529 \\
0.053\end{array}$ \\
\hline iCOR & 0.86 & 0.004 & 0.056 & 30 & $\begin{array}{l}\mathrm{Y}=0.980 \mathrm{x} \\
0.038\end{array}$ \\
\hline
\end{tabular}

HohenpeissenbergDWD Station 


\begin{tabular}{lllllll}
\hline MAJA & 0.64 & 0.007 & 0.058 & 32 & $\begin{array}{l}\text { Y }= \\
0.080\end{array}$ & $0.525 \mathrm{x}^{+}$ \\
\hline
\end{tabular}
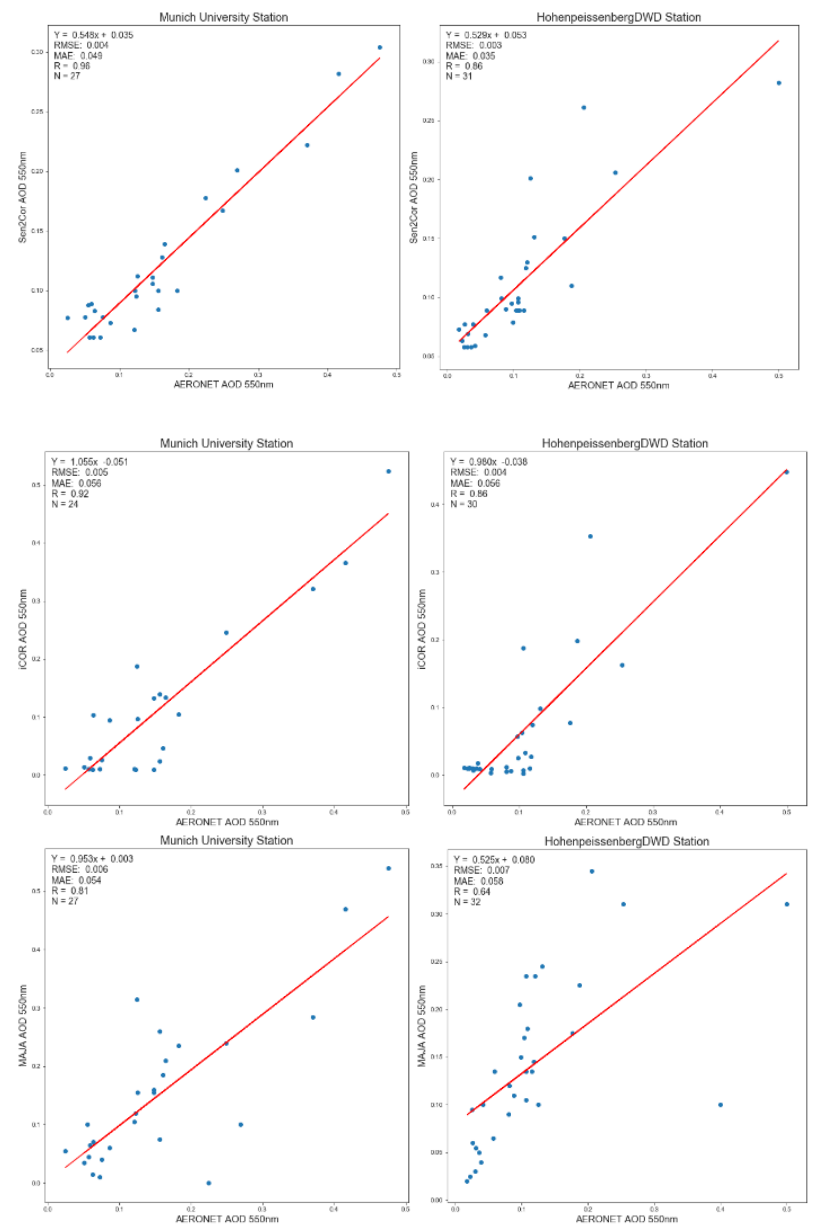

Figure 3: Scatterplots and regression lines between Sentinel -2 satellite retrieved AOD and AERONET AOD from Munich University and HohenpeissenbergDWD stations.

\section{Spatial and Temporal Variability}

The satellite retrieved AODs were further compared against the AERONET AOD over time to study the temporal trends as shown in Figure 4. In both locations, peaks in AOD levels are clearly distinctive for example in June and August. However, while the AOD retrievals for Munich University station display a smooth trend that correlates well with the AERONET AOD, in HohenpeissenbergDWD station, the retrievals display erratic behaviour and larger deviations from the AERONET stations due to the unstable surface reflectances.
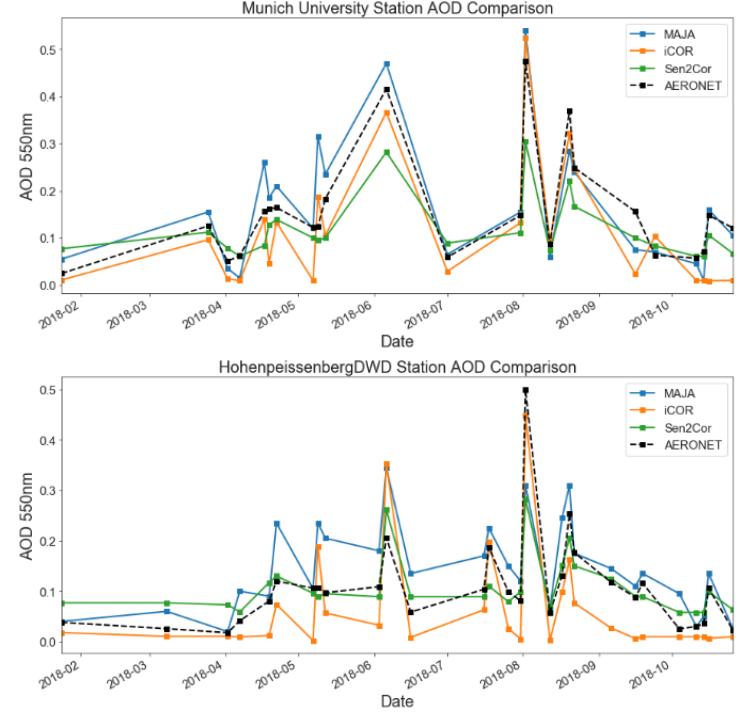

Figure 4: Timeseries charts of Sentinel -2 AOD retrievals using Sen2Cor, iCOR and MAJA algorithms against AERONET AOD

Despite Sen2Cor and iCOR algorithm achieving a high agreement with AEORONET AOD, the output maps generalize AOD distributions failing to show spatial variations especially in smaller regions such as the Munich City region Figure 5(a, b). On the other hand, AOD retrieved using MAJA algorithm, shows boundaries between slight variations in AOD providing better mapping in smaller regions.

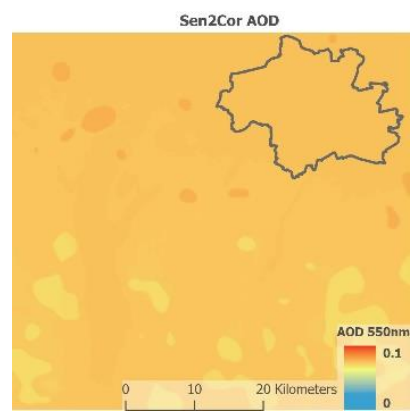

(a)

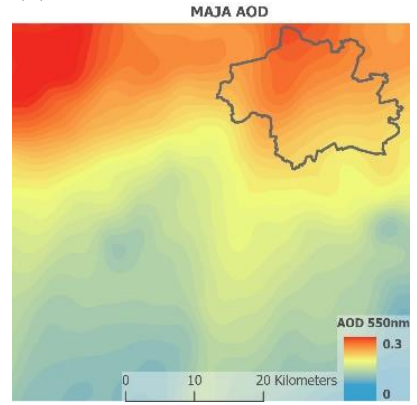

(c)

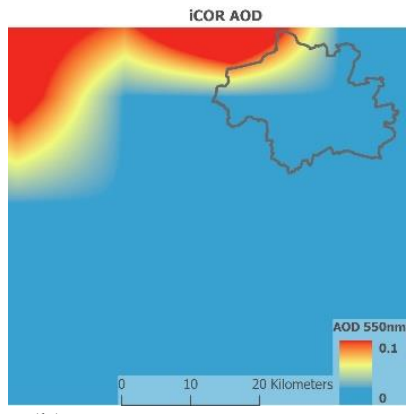

(b)

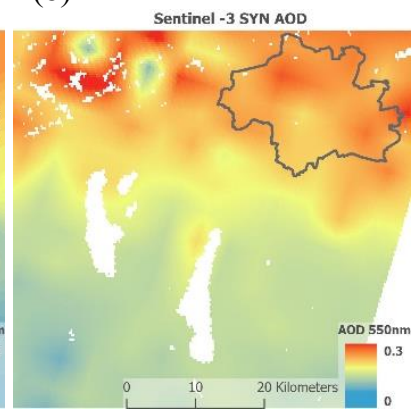

(d)

Figure 5: Figures (a), (b) and (c) show maps of AOD retrieved from Sentinel -2 images using Sen2Cor, iCOR and MAJA algorithms respectively, while figure (d) shows the AOD map from Sentinel -3 SYN product. The images were acquired on $26^{\text {th }}$ October 2018, at 10:11 am for Sentinel -2 and 10:09 am for Sentinel -3 . 
In Figure 5(c) higher AOD concentrations are visible in the northern part of the city, with gradual decrease towards the southern rural region as expected due to the concentration of anthropogenic activities in the urban region. Comparing the maps with the readily available Sentinel -3 SYN AOD product Figure 5(d), MAJA AOD map is the most similar in both AOD distribution and levels. Conversely, Sen2Cor and iCOR algorithms retrieve lower AOD values with no matching distribution patterns to the Sentinel -3 SYN AOD. iCOR retrieved AOD appears in rectangular patterns which is a result of subdivision into $15 \times 15 \mathrm{~km}$ tiles during the AOD retrieval phase and the mosaicking thereafter in the postprocessing step. The restriction of AOD boundaries per tile results to sharp boundaries between tiles as shown in Figure 5(b).

\section{SARA Algorithm}

AOD retrieved by the algorithm had no correlation with the AERONET AOD data and thus was omitted from the statistical analysis. AOD retrieval shows high dependence on BOA reflectances. In the study area, brighter surfaces produced higher AOD values compared to darker areas. This results in an AOD map that highly correlates with the BOA reflectances. Visually inspecting the map in Figure 6, built-up areas and road surfaces have higher AOD values while darker areas with vegetation cover have lower values. The assumption that aerosol properties, single scattering albedo and asymmetry factor, are constant over a region is also not met in our study area.

The aerosol properties obtained from the two AERONET stations, which are approximately $57 \mathrm{~km}$ apart, differ in varying magnitudes over space and time. Aerosols distribution and properties vary considerably over space and time which limits the application of the algorithm in our study area. This is evident from measurements recorded by the two AERONET stations.

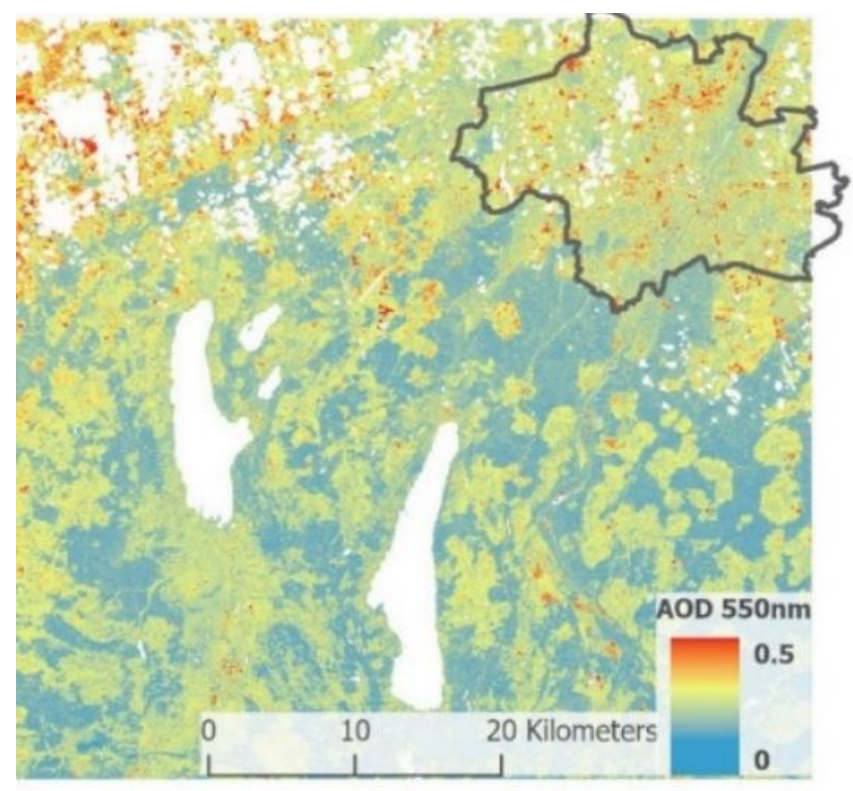

Figure 6: SARA retrieved AOD for Sentinel -2 image acquired on $26^{\text {th }}$ October 2018

\section{AOD-PM 2.5 estimation}

Sentinel -3 SYN AOD which is readily processed and shows a high agreement with AERONET AOD is ideal for rapid mapping of air quality. The pair of Sentinel-3 satellites also have a high temporal resolution with a short revisit time of less than two days. As shown in Figure 5 (d) the AOD product also captures spatial variability of aerosols at $300 \mathrm{~m}$ spatial resolution. We used multiple linear regression with this AOD and meteorological data to estimate $\mathrm{PM}_{2.5}$ for the Stuttgart City study area. Table 2 shows the data, excluding outliers and null values, used for the regression analysis. $80 \%$ of the dataset was used to train the regression models while $20 \%$ was used to test predictions from the models.

Table 2: Descriptive statistics of the dataset used in regression analysis.

\begin{tabular}{llllllll} 
& PM2.5 & AOD & $\begin{array}{l}\text { Temp } \\
\left(\mathbf{C}^{\circ}\right)\end{array}$ & $\begin{array}{l}\text { RH } \\
(\%)\end{array}$ & $\begin{array}{l}\text { P } \\
(\mathbf{h P a})\end{array}$ & $\begin{array}{l}\text { WS } \\
(\mathbf{m} / \mathbf{s})\end{array}$ & $\begin{array}{l}\text { WD } \\
(\mathbf{d e g})\end{array}$ \\
\hline Count & 1451 & 1451 & 1451 & 1451 & 1451 & 1451 & 1451 \\
\hline Mean & 4.36 & 0.48 & 27.4 & 45.44 & 1021.8 & 2.79 & 143.3 \\
\hline Std & 3.02 & 0.11 & 2.95 & 9.22 & 1.56 & 0.9 & 116.5 \\
\hline Min & 0 & 0.21 & 22.25 & 37 & 1020 & 1.49 & 70 \\
\hline $\mathbf{2 5 \%}$ & 2.56 & 0.4 & 25.35 & 38 & 1020 & 2.1 & 80 \\
\hline $\mathbf{5 0 \%}$ & 3.43 & 0.45 & 27.81 & 42 & 1022 & 2.6 & 82.87 \\
\hline $\mathbf{7 5 \%}$ & 4.91 & 0.56 & 29.41 & 56 & 1023 & 3.48 & 95 \\
\hline Max & 18.32 & 0.82 & 31.86 & 60 & 1024 & 4.1 & 360 \\
\hline
\end{tabular}

From the regression analysis, PM showed a positive correlation with $\mathrm{AOD}$, relative humidity $(\mathrm{RH})$, wind speed (WS) and atmospheric pressure (P) while temperature and the wind direction (WD) showed negative correlation as shown on Table 3. 
Table 3: Regression Coefficients

\begin{tabular}{lr} 
Independent Variables & \multicolumn{1}{l}{$\begin{array}{l}\text { Regression } \\
\text { Coefficients }\end{array}$} \\
\hline AOD & 0.41335 \\
\hline Temperature & -0.1339 \\
\hline Relative Humidity & 0.033356 \\
\hline Wind Speed & 2.586447 \\
\hline Wind Direction & -0.02118 \\
\hline Pressure & 3.052675 \\
\hline
\end{tabular}

As expected, the columnar AOD has a significant positive correlation to the PM. The wind speed and direction play a significant role in the dispersion and concentration of PM. Due to the geographic location of our study area, where the city lies in a valley, airflow could be inhibited depending on the wind direction and speed. Wind speeds and direction are uniform spatially and only vary over time. Based on the elevation map of Stuttgart in Figure 7 (a), winds blowing from between 0 90 degrees are bound to promote deposition of PM which is trapped in the lower altitude regions. As the wind direction increases, PM is dispersed away from the city region and hence the weak negative correlation.

The atmospheric pressure, which is dependent on the elevation and ambient temperature, has a significant influence on the surface-level PM concentrations. In the study area, atmospheric pressure is uniform spatially and only varies with time due to temperature differences. The predicted $\mathrm{PM}_{2.5}$ concentrations exhibited agreement with ground measurements, with an overall coefficient $\left(\mathrm{R}^{2}\right)$ of 0.59 .

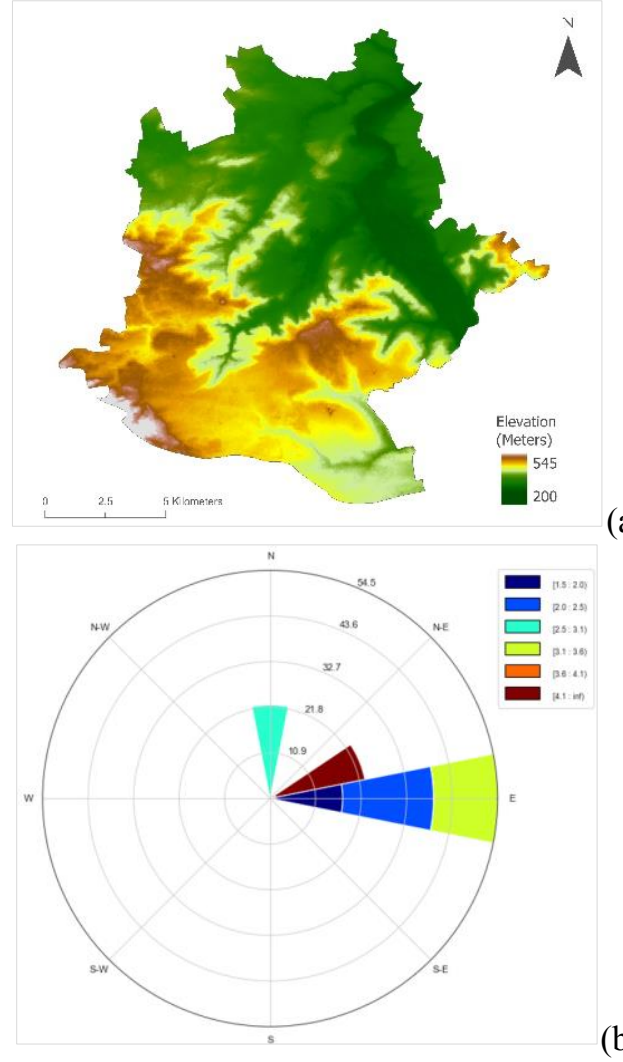

Figure 7: (a) Elevation of Stuttgart City region derived from SRTM DEM and (b) A wind rose plot showing the normalized frequency of wind data used in the study.

\section{Conclusions}

The study used different algorithms to retrieve AOD from Sentinel -2 images and evaluates their accuracies against AERONET measured AOD. While Sen2Cor and iCOR algorithms achieve better correlations with AERONET AOD for both stations, they fail to capture detailed spatial variations in AOD distribution. MAJA algorithm on the hand achieves lower correlations for both stations but shows detailed variations of AOD spatially. Though at a coarser spatial resolution of $300 \mathrm{~m}$ and no data pixels due to masking out clouds, cloud shadows, snow and water bodies, Sentinel -3 SYN AOD product matches MAJA retrieved AOD and slight spatial variations in AOD values are visible. The algorithms also show the ability to identify temporal trends in AOD values similar to the ground measurements from AERONET stations. BOA reflectances are significant in AOD retrieval with stable surfaces such as in built-up surfaces in the urban region showing better agreements with AERONET AOD while in rural regions where the vegetation changes rapidly over time, the retrieved AOD shows lesser agreement for the three algorithms.

The DDV approach as used in Sen2Cor does not provide detailed spatial variation of AOD. Conversely, not relying on prior knowledge of BOA reflectances such as is the case with the implementation of the multispectral approach in MAJA and the multi-view-angle approach in Sentinel -3 SYN, gives a better spatial variation of AOD distribution. Sentinel -2 AOD retrievals show strong agreement with ground measured AOD and potential to better map aerosols in urban environments. In this study, MAJA algorithm produced the best detailed spatial variations at $10 \mathrm{~m}$ spatial resolution. At $300 \mathrm{~m}$ spatial resolution, Sentinel -3 SYN AOD also identifies variations at a local scale and could be used for aerosol monitoring in urban environments.

Satellite retrieved AOD offers the ability to estimate surface level PM concentrations allowing full spatial extent mapping of air pollution. One of the limitations of using sun-synchronous satellites like Sentinel -3 is that they pass over a target area at the same local mean solar time, approximately 10:00 GMT over Stuttgart. This limits them from being used to estimate diurnal variations of air quality. The approach used in the study can be further improved to model diurnal, weekly and seasonal AOD, PM and meteorological factors relationships.

\section{Acknowledgements}

The project ,__city: intelligent city“is sponsored by the Federal Ministry of Education and Research (BMBF) under the promotion code 13FH9I011A and supervised by 
the project executing organization VDI

Technologiezentrum GmbH for the BMBF.

Publication is funded by the Polish National Agency for Academic Exchange under the International Academic Partnerships Programme from the project „Organization of the 9th International Scientific and Technical Conference entitled Environmental Engineering, Photogrammetry, Geoinformatics - Modern Technologies and Development Perspectives".

\section{References}

1. United Nations. World Urbanization Prospects: The 2018 Revision, New York, 2019. Available online: https://population.un.org/wup/ (accessed on 8 February 2019).

2. Kaufman, Y.J.; Sendra, C. Algorithm for automatic atmospheric corrections to visible and near-IR satellite imagery. International Journal of Remote Sensing 1988, 9, 1357-1381, doi:10.1080/01431168808954942.

3. Kaufman, Y.J.; Wald, A.E.; Remer, L.A.; Gao, B.C.; Li, R.-R.; Flynn, L. The MODIS 2.1- um Channel-Correlation with Visible Reflectance for Use in Remote Sensing of Aerosol. IEEE Trans. Geosci. Remote Sensing 1997, 35, 1286-1298, doi:10.1109/36.628795.

4. Gillingham, S.S.; Flood, N.; Gill, T.K.; Mitchell, R.M. Limitations of the dense dark vegetation method for aerosol retrieval under Australian conditions. Remote Sensing Letters 2012, 3, 67-76, doi:10.1080/01431161.2010.533298.

5. Hagolle, O.; Huc, M.; Villa Pascual, D.; Dedieu, G. A Multi-Temporal and Multi-Spectral Method to Estimate Aerosol Optical Thickness over Land, for the Atmospheric Correction of FormoSat-2, LandSat, VEN $\mu \mathrm{S}$ and Sentinel-2 Images. Remote Sensing 2015, 7, 2668-2691, doi:10.3390/rs70302668.

6. Hagolle, O.; Hug, M.; Desjardins, C.; Auer, S.; Richter, R. Maja Algorithm Theoretical Basis Document, 2017. Available online: https://doi.org/10.5281/zenodo.1209633 (accessed on 8 February 2019).

7. Remer, L.A.; Mattoo, S.; Levy, R.C.; Munchak, L.A. MODIS $3 \mathrm{~km}$ aerosol product: Algorithm and global perspective. Atmos. Meas. Tech. 2013, 6, 1829-1844, doi:10.5194/amt-6-1829-2013.

8. Wu, Y.; Graaf, M. de; Menenti, M. Improved MODIS Dark Target aerosol optical depth algorithm over land: Angular effect correction. Atmos. Meas. Tech. 2016, 9, 5575-5589, doi:10.5194/amt-9-5575-2016.

9. North, P.; Heckel, A. Sentinel-3 Optical Products and Algorithm Definition. SYN Algorithm Theoretical Basis Document No. 2.3, 2010. Available online: https://earth.esa.int/documents/247904/349589/SY N_L2-3_ATBD.pdf (accessed on 8 March 2019).
10. Giles, D.M.; Sinyuk, A.; Sorokin, M.G.; Schafer, J.S.; Smirnov, A.; Slutsker, I.; Eck, T.F.; Holben, B.N.; Lewis, J.R.; Campbell, J.R.; et al.

Advancements in the Aerosol Robotic Network (AERONET) Version 3 database - automated nearreal-time quality control algorithm with improved cloud screening for Sun photometer aerosol optical depth (AOD) measurements. Atmos. Meas. Tech. 2019, 12, 169-209, doi:10.5194/amt-12-169-2019.

11. Main-Knorn, M.; Pflug, B.; Louis, J.; Debaecker, V.; Müller-Wilm, U.; Gascon, F. Sen2Cor for Sentinel-2. In Image and Signal Processing for Remote Sensing XXIII, 2017; p 1042704.

12. Louis, J.; Debaecker, V.; Pflug, B.; Main-Korn, M.; Bieniarz, J.; Mueller-Wilm, U.; Cadau, E.; Gascon, F. Sentinel-2 Sen2Cor: L2A Processor for Users. In Living Planet Symposium, 2016; p 91.

13. Richter, R.; Louis, J.; Berthelot, B. Sentinel-2 MSILevel 2 A Products Algorithm Theoretical Basis Document Issue 1.8, 2011. Available online: https://earth.esa.int/c/document_library/get_file?fol derId $=349490 \&$ name $=$ DLFE-4 $\overline{5} 18$.pdf (accessed on 8 February 2019).

14. Keukelaere, L. de; Sterckx, S.; Adriaensen, S.; Knaeps, E.; Reusen, I.; Giardino, C.; Bresciani, M.; Hunter, P.; Neil, C.; van der Zande, D.; et al. Atmospheric correction of Landsat-8/OLI and Sentinel-2/MSI data using iCOR algorithm: Validation for coastal and inland waters. European Journal of Remote Sensing 2018, 51, 525-542, doi:10.1080/22797254.2018.1457937.

15. Guanter, L.; Alonso, L.; Moreno, J. First results from the PROBA/CHRIS

hyperspectral/multiangular satellite system over land and water targets. IEEE Geoscience and Remote Sensing Letters 2005, 2, 250-254, doi:10.1109/LGRS.2005.851542.

16. Guanter Palomar, L. New algorithms for atmospheric correction and retrieval of biophysical parameters in Earth Observation. Application to ENVISAT/MERIS data; Universitat de València, 2007, ISBN 9788469307618.

17. Bilal, M.; Nichol, J.E.; Bleiweiss, M.P.; Dubois, D. A Simplified high resolution MODIS Aerosol Retrieval Algorithm (SARA) for use over mixed surfaces. Remote Sensing of Environment 2013, 136, 135-145, doi:10.1016/j.rse.2013.04.014.

18. Qiu, S.; Zhu, Z.; He, B. Fmask 4.0: Improved cloud and cloud shadow detection in Landsats 4-8 and Sentinel-2 imagery. Remote Sensing of Environment 2019, 231, 111205, doi:10.1016/j.rse.2019.05.024.

19. Baetens, L.; Desjardins, C.; Hagolle, O. Validation of Copernicus Sentinel-2 Cloud Masks Obtained from MAJA, Sen2Cor, and FMask Processors Using Reference Cloud Masks Generated with a Supervised Active Learning Procedure. Remote Sensing 2019, 11, 433, doi:10.3390/rs11040433.

20. Rahman, H.; Verstraete, M.M.; Pinty, B. Coupled surface-atmosphere reflectance (CSAR) model: 1. Model description and inversion on synthetic data. 
J. Geophys. Res. 1993, 98, 20779, doi:10.1029/93JD02071.

21. Vermote, E.F.; Tanre, D.; Deuze, J.L.; Herman, M.; Morcette, J.-J. Second Simulation of the Satellite Signal in the Solar Spectrum, 6S: An overview. IEEE Trans. Geosci. Remote Sensing 1997, 35, 675-686, doi:10.1109/36.581987.

22. Bodhaine, B.A.; Wood, N.B.; Dutton, E.G.; Slusser, J.R. On Rayleigh Optical Depth Calculations. J. Atmos. Oceanic Technol. 1999, 16, 1854-1861, doi:10.1175/1520 0426(1999)016<1854:ORODC >2.0.CO;2.

23. Berberan-Santos, M.N.; Bodunov, E.N.; Pogliani, L. On the barometric formula. American Journal of Physics 1997, 65, 404-412, doi:10.1119/1.18555.

24. Levy, R.C.; Remer, L.A.; Mattoo, S.; Vermote, E.F.; Kaufman, Y.J. Second-generation operational algorithm: Retrieval of aerosol properties over land from inversion of Moderate Resolution Imaging Spectroradiometer spectral reflectance. J. Geophys. Res. 2007, 112, doi:10.1029/2006JD007811.

25. Liu, F.; Tan, Q.-W.; Jiang, X.; Jiang, W.-J.; Song, D.-L. Effect of Relative Humidity on Particulate Matter Concentration and Visibility During Winter in Chengdu. Huan Jing Ke Xue. 2018, 39, 14661472, doi:10.13227/j.hjkx.201707112.

26. Munir, S.; Habeebullah, T.M.; Mohammed, A.M.F.; Morsy, E.A.; Rehan, M.; Ali, K. Analysing PM2.5 and its Association with PM10 and Meteorology in the Arid Climate of Makkah, Saudi Arabia. Aerosol Air Qual. Res. 2017, 17, 453-464, doi:10.4209/aaqr.2016.03.0117.

27. Li, Y.; Chen, Q.; Zhao, H.; Wang, L.; Tao, R. Variations in PM10, PM2.5 and PM1.0 in an Urban Area of the Sichuan Basin and Their Relation to Meteorological Factors. Atmosphere 2015, 6, 150163, doi:10.3390/atmos6010150. 